\title{
CD8+ $T$ cell immune response against non-viral pathogens
}

\author{
Imtiaz A. Khan ${ }^{1}$
}

Received: 6 April 2015 / Accepted: 6 April 2015 /Published online: 6 May 2015

(C) Springer-Verlag Berlin Heidelberg 2015

$\mathrm{CD} 8+\mathrm{T}$ cells are important for the protective immunity against intracellular infections [1]. These cells can be an important source of inflammatory cytokines like IFN $\gamma, \mathrm{TNF} \alpha$ and IL-2 [2], considered important for host protection, and can also directly control the spread of pathogens by exhibiting cytolytic activity against infected cells [3]. During early stages, when mysteries of adaptive immunity had started to unravel, CD8 T cells were named as CTLs (cytotoxic T cells) that were primarily thought to be involved in killing of virally infected cells. However, with the passage of time, it was discovered that in addition to their cytolytic function, CD8+ T cells, like CD4+ T cell population, possessed the ability to secrete important inflammatory cytokines. However, it was generally believed that their cytotoxic function was restricted to viral pathogens, where they played a critical role in keeping the infection under control.

Over the years, the role of CD8 T cells has been extended to non-viral infections like Listeria monocytogenes, Plasmodium species, Trypanosoma cruzi and Toxoplasma gondii where they have been reported to play a central role in host protection. Information related to the generation and maintenance of robust and functional CD8 T cell responses against these pathogens is key for strategizing the development of immunotherapeutic agents against these diseases. For Plasmodium infection, maintenance of effector and memory CD8+ T cells

This article is a contribution to the Special Issue on: $\mathrm{CD} 8^{+} \mathrm{T}$-cell Responses against Non-Viral Pathogens - Guest Editors: Fidel Zavala and Imtiaz A. Khan

Imtiaz A. Khan

imti56@gwu.edu

1 Department of Microbiology, Immunology and Tropical Medicine, George Washington University, Washington, DC 20037, USA especially against liver stage parasite is a major challenge for developing a successful vaccine against this pathogen, which causes severe complications in large number of human population [4, 5]. Control of chronic toxoplasmosis is predominantly dependent on multifunctional CD8 T cells and protective epitopes responsible for the maintenance of this response need to be identified [6]. Amongst the non-viral infections, intracellular bacteria, L. monocytogenes, has served as a model pathogen that has contributed immensely to the information related to generation and differentiation of memory CD8 $\mathrm{T}$ cell responses [7, 8]. T. cruzi infection, which causes Chagas disease in humans, is one of the important protozoan parasites where CD8 T cells play a dominant role in host protection [9]. However, in spite of effective CD8+ T cell immune responses generated against $T$. cruzi, the host fails to clear the infection, which has been attributed to late expressing parasite epitopes [10]. In this situation, it is very important to identify and adopt the measures that will result in the amplification of CD8 T cell immunity, needed to clear this infection. Although polyfunctional CD4 T cells were targeted for vaccination against Mycobacterium tuberculosis, the strategy did not yield desired results [11]. A review article on this subject in this issue suggests the inclusion of CD8 T cells specific for mycobacterial antigens in the immunization protocols. In addition to these non-viral pathogens where CD8 $\mathrm{T}$ cells play a pivotal role in host protection, the issue also deals with infections caused by Leishmania (another protozoan parasite) [12] or microsporidia (fungal infection) [13], where CD8 T cells play an important synergistic role.

As stated above, reviews related to viral infections which discuss the elicitation and maintenance of CD8+ T cell immunity against these pathogens are available. Although the information about CD8 $\mathrm{T}$ cell immunity against non-viral pathogens has been accumulating, reviews dedicated to the subject are at best very limited. It is very timely that information about 
CD8 T cell responses against these pathogens be discussed in detail and challenges facing to develop robust and sustainable immunity in the infected host identified. The approaches needed to achieve persistent and functional CD8 $\mathrm{T}$ cell responses against these complex microbial agents could be different from those required for viral infections. The articles in this issue have been contributed by leading researchers in the field and we hope the readers find it informative and interesting.

\section{References}

1. Appay V, Douek DC, Price DA (2008) CD8+ T cell efficacy in vaccination and disease. Nat Med 14(6):623-8

2. Kristensen NN et al (2004) Cytokine production by virus-specific $\mathrm{CD} 8(+) \mathrm{T}$ cells varies with activation state and localization, but not with TCR avidity. J Gen Virol 85(Pt 6):1703-12

3. Demers KR, Reuter MA, Betts MR (2013) CD8(+) T-cell effector function and transcriptional regulation during HIV pathogenesis. Immunol Rev 254(1):190-206

4. Tse SW, Radtke AJ, Zavala F (2011) Induction and maintenance of protective $\mathrm{CD} 8+\mathrm{T}$ cells against malaria liver stages: implications for vaccine development. Mem Inst Oswaldo Cruz 106(Suppl 1): $172-8$

5. Cockburn IA et al (2010) Prolonged antigen presentation is required for optimal CD8+ T cell responses against malaria liver stage parasites. PLoS Pathog 6(5), e1000877

6. Bhadra R, Gigley JP, Khan IA (2011) The CD8 T-cell road to immunotherapy of toxoplasmosis. Immunotherapy 3(6):789-801

7. Condotta SA et al (2012) Probing CD8 T cell responses with Listeria monocytogenes infection. Adv Immunol 113:51-80

8. Martin MD et al (2012) Population dynamics of naive and memory $\mathrm{CD} 8 \mathrm{~T}$ cell responses after antigen stimulations in vivo. J Immunol 188(3):1255-65

9. Padilla AM, Bustamante JM, Tarleton RL (2009) CD8+ T cells in Trypanosoma cruzi infection. Curr Opin Immunol 21(4):385-90

10. Padilla AM, Simpson LJ, Tarleton RL (2009) Insufficient TLR activation contributes to the slow development of CD8+ T cell responses in Trypanosoma cruzi infection. J Immunol 183(2): $1245-52$

11. Tameris MD et al (2013) Safety and efficacy of MVA85A, a new tuberculosis vaccine, in infants previously vaccinated with BCG: a randomised, placebo-controlled phase $2 \mathrm{~b}$ trial. Lancet 381(9871): $1021-8$

12. Kaye P, Scott P (2011) Leishmaniasis: complexity at the hostpathogen interface. Nat Rev Microbiol 9(8):604-15

13. Moretto M, Weiss LM, Khan IA (2004) Induction of a rapid and strong antigen-specific intraepithelial lymphocyte response during oral encephalitozoon cuniculi infection. J Immunol 172(7):4402-9 